\title{
„Russisches Roulette“ in der Genforschung am Menschen?
}

\author{
Bettina Schöne-Seifert
}

Online publiziert: 9. Januar 2019

(C) Springer-Verlag GmbH Deutschland, ein Teil von Springer Nature 2019

\section{Vorbemerkungen}

Genforschung wird seit einigen Jahren zunehmend durch das sogenannte GenomEditieren bestimmt. Hierbei kommen „Genscheren“ zum Einsatz: CRISPR \& Co, darunter neuerdings auch schonendere Instrumente, die ohne ein Zerschneiden des DNA-Doppelstrangs arbeiten (Gaudelli et al. 2017). Mit Hilfe dieser Methodik lassen sich Genfunktionen aller Lebewesen verändern, indem gezielt molekulare „Buchstaben“ (Basen) ihrer Genome entfernt oder ersetzt werden.

Im letzten Editorial, das ich für diese Zeitschrift, im Frühjahr 2017, schreiben durfte, ging es um die Frage der ethischen Zulässigkeit forschender Genom-Editierung an der menschlichen Keimbahn. Damals hatte soeben die weltweit erste (chinesische) Publikation über durchgeführtes menschliches „GLGE“, wie es international für germ-line genome editing steht, globale Kontroversen ausgelöst (Tang et al. 2017). Dabei hatten die Forscher CRISPR-Cas9 dazu eingesetzt, an sehr frühen und zudem nicht fortpflanzungsfähigen Embryonen in der Glasschale versuchsweise einen schweren Gendefekt zu reparieren. Der Versuch gelang partiell und belegte so zum einen die damals noch sehr hohe Fehlerquote des Eingriffs, zum anderen aber auch dessen grundsätzliche Machbarkeit.

\section{Der Frevel des Dr. He}

Kürzlich nun, Ende November 2018, hat erneut ein angeblich in China vorgenommenes GLGE-Forschungsprojekt Wellen geschlagen - diesmal allerdings ausschließlich Schockwellen: In rasender Geschwindigkeit verbreitete sich über die digitalen

Prof. Dr. B. Schöne-Seifert $(\bowtie)$

Institut für Ethik, Geschichte und Theorie der Medizin, Universität Münster,

Von-Esmarch-Str. 62, 48149 Münster, Deutschland

E-Mail: schoeneb@ukmuenster.de 
Medien millionenfaches Entsetzen darüber, dass der 34-jährige chinesische Genforscher Jiankui He nach eigenen Angaben die Geburt eines CRISPR-Zwillingspaares verantwortet hat. Er behauptet, bei ihnen ein Gen blockiert zu haben, das HIV-Infektionen ermöglicht, und veröffentlichte seine Meldung werbewirksam am Vorabend des Zweiten Internationalen Gipfeltreffens zur Genom-Editierung in Honkong. Zum Zeitpunkt, zu dem ich dies schreibe, gilt Dr. He als untergetaucht oder unter Arrest gestellt (vgl. Epoch Times 2018) und es bleiben so gut wie alle Einzelheiten über Faktizität, Ablauf und Motivation, Hintermänner und Strafwürdigkeit der Erzeugung der angeblichen CRISPR-Babys zu klären. Einmal abgesehen von berechtigter massiver Kritik an zahlreichen prozeduralen Aspekten des Vorgehens (von der Indikationsstellung bis zur Aufklärung), die von vielen Seiten aufgearbeitet worden ist (exemplarisch Young 2018), speist sich das Entsetzen diesmal vor allem aus zwei Quellen:

Grob skizziert sehen die einen den primären Tabubruch darin, dass mit den angeblich gen-editierten Zwillingen eine kategorisch verbotswürdige Manipulation des „menschlichen Genpools“ vollzogen worden sei. Die anderen hingegen halten diesen „Genpool“ keineswegs für sakrosankt, wenn es um die Umkehr pathogener Mutationen geht. Gerade deshalb sind sie doppelt entsetzt über das eigenmächtige Vorpreschen des chinesischen Youngsters. Angesichts noch ungeklärter Risiken habe er nicht nur die konkreten Babys gefährdet, sondern auch die Kontrollierbarkeit der Gentechnik und damit deren künftige Förderung und Akzeptanz trotz großer klinischer Nutzenpotentiale prinzipiell in Frage stellt. Diese zweite Position vertraten bereits auf dem Hongkong-Gipfel die organisierenden Institutionen, nämlich der Wissenschaftsakademien der USA, Großbritanniens und Hongkongs. Und seither gab es einige Verstärkung (beispielhaft: Dzau et al. 2018). Im Folgenden möchte auch ich eindringlich davor warnen, Dr. He als Totengräber der GLGE- oder gar der gesamten humanen Gentechnik-Forschung zu verstehen, wie es in Deutschland nicht wenige Meinungsführer zu tun geneigt scheinen.

$\mathrm{Zu}$ dieser fundamental-ablehnenden Haltung, deren Ausmaß sich derzeit nur erahnen lässt, trägt offenbar auch ein gerütteltes $\mathrm{Ma} \beta$ an genetischer Unbildung bei. In Deutschland, so zeigten auch in Hongkong Vergleichsdaten der UK-Sozialwissenschaftlerin Anna Middleton, steht es in dieser Hinsicht besonders schlecht (vgl. Middleton et al. 2018). Hier bleibt zunächst auf dem Bildungssektor einiges zu tun. Und nun zu Sachstands- und Bewertungsfragen der Gentechnik.

\section{Somatische Gentherapien in der Erprobung}

Eine erste Kategorie von Genreparatur kann auf der Ebene nicht-vererbbarer, fehlfunktionierender Spezialzellen stattfinden. Deren jeweilige Vorläuferzellen, so das Behandlungsprinzip, werden den Patienten entnommen, gen-editiert und wieder eingesetzt. Auf diesem technisch wie ethisch vergleichsweise unproblematischen Gebiet ist gegenwärtig - dauer-pessimistische Gentherapie-Kritiker Lügen strafend - das erste Dutzend therapeutischer Ansätze in der experimentellen klinischen Erprobung. Und es werden absehbar mehr. Es geht etwa um die Behandlung von Hämoglobinopathien, tödlichem Muskelschwund sowie Blut- oder Lungenkrebs. Für einen Be- 
handlungserfolg, so zeigen die Daten, reicht es dabei oft aus, wenn über die besagte Reparatur eine Minderheit der Spezialzellen im Körper wieder normal funktioniert. Über Aufwand und Nachhaltigkeit solcher Eingriffe weiß man noch zu wenig. Chinesische Forscher jedenfalls liegen bei den klinischen Studien in Führung vor den USA.

\section{Grundlagenforschung an Keimbahnzellen}

Die ersten Teilungen einer befruchteten menschlichen Eizelle im Video-Zeitraffer zu beobachten, schon das beeindruckt selbst Hartgesottene. Noch viel eindrucksvoller ist es, dabei verfolgen zu können, wie das gezielte Ausschalten eines einzelnen Gens früheste Entwicklungsschritte hemmt und dabei völlig anders wirkt als etwa in einem Maus-Embryo. Aus solchen Untersuchungen, wie sie nun mithilfe von CRISPR durchgeführt werden, könnten grundlegende Einsichten zur Verbesserung von assistierter Befruchtung (80.000 IVF-Versuche pro Jahr allein in Deutschland) und zur Therapie bisher unverstandener Krankheiten erwachsen.

Großbritannien, Belgien oder Israel etwa erlauben es, zu Forschungszwecken menschliche Embryonen herzustellen und zu kultivieren (für maximal 14 Tage: dann sind sie knapp 0,2 mm große Formationen). Diese Staaten sind hier ,,im Vorteil“ gegenüber den Ländern, die Forschung nur an verwaisten IVF-Embryonen zulassen (etwa Frankreich oder Japan) oder die Embryonenforschung gänzlich untersagen (wie Deutschland, Portugal oder Irland). Selbstredend werden wissenschaftliche Vorteile durch ethische Argumente übertrumpft. Aber es gibt in dieser unendlichen Debatte keine vernünftig-zwingenden Argumente für die Schutzwürdigkeit sehr früher Embryonen. Vielmehr ist hier auf biopolitischer Ebene dem pragmatischen Respektieren eines ethischen Pluralismus das Wort zu reden. Forschung an frühen Embryonen sollte ins Ermessen der konkreten Spender, Forscher und Geldgeber gestellt werden. Deutschland drückt sich um diese Debatte und ihre Ent-Dogmatisierung.

\section{Perspektiven auf die Keimbahn-Reparatur}

Ungleich risikobehafteter als somatisches Gen-Editieren sind bekanntlich Reparaturen am Erbgut von Spermien oder von (un)befruchteten Eizellen. Hier nämlich könnten ungewollte Fehler nicht nur alle Zellen des sich entwickelnden Organismus betreffen, sondern sie würden sich zudem auch auf Kinder und Kindeskinder vererben. Unerlässlich wäre daher die Gewissheit, keine Nebeneffekte auf die DNABasenabfolge oder auf das Zusammenspiel von Genen untereinander und mit der Umwelt auszulösen. Das hier erforderliche Maß an vorhersagbarer Präzision schien und scheint vielen noch heute unerreichbar. Doch mehren sich auch optimistischere Stimmen. Mit verfeinerten Editierungs-Techniken und multiplen Qualitätstests an Zelllinien aus experimentell veränderten Embryonen meinen manche, eines Tages hinreichende Sicherheit gewinnen zu können. Was hier machbar und realistisch ist, bleibt abzuwarten. Ganz gewiss jedenfalls verbieten bestehende Forschungslücken, fehlende Risikostandards und zahllose andere „Fragezeichen“ bis auf weiteres jeg- 
liche GLGE-Anwendung in der Klinik. Schon daher war Hes angebliche CRISPRSchwangerschaft unverantwortlich.

Angesichts der schwierigen Risikobeurteilung wird für einen potentiellen Einsatz des Keimbahn-Editierens in der Klinik zudem gefordert, dass er schwere, bisher unbehandelbare Erkrankungen betreffen und ohne akzeptable Alternative sein müsse - auch das war Bestandteil eines Hongkong-Konsenses. Allerdings ist die Beurteilung von Alternativen, etwa Kinderlosigkeit oder Adoption, sehr subjektiv. Faktisch wäre bei monogenen Erkrankungen zumeist auch die Methode der Präimplantationsdiagnostik eine Alternative, die allerdings als Selektionsmaßnahme nicht von allen Betroffenen ethisch akzeptiert wird (und die in Deutschland auch nur in engen Ausnahmefällen legal ist). Zudem rücken neuerdings auch polygene Krankheiten in den Bereich möglicher zukünftiger Behandelbarkeit. Wo vergleichende GenomStudien (GWAS) von riesigem Umfang zunehmend zeigen, welche Genveränderungen erst im multifaktoriellen Zusammenspiel krankmachen, steigen die Hoffnungen, eines Tages auch schwerste Beeinträchtigungen auf polygener Ebene erfolgreich zu behandeln. Hier böte PID keine Alternative.

Auf dem Hongkong-Gipfel brachte das Abschlusskommuniqué des Organisationskomitees diesen vorsichtig-liberalen Optimismus zum Ausdruck (Baltimore et al. 2018). Darin wird sogar bereits ein hypothetischer Pathway für die klinische Erprobung heilender Keimzell-Eingriffe angeregt. Dieser Tenor verwundert kaum, denn einen ähnlichen Tenor hatten schon die beiden bedeutendsten publizierten EthikStellungnahmen zu GLGS: diejenige der US-Akademien von 2017 (National Academies of Scienes, Engineering, and Medicine 2017) und diejenige (zweite) des britischen Nuffield-Council vom Juli 2018 (Nuffield Council 2018); mehrere Autoren dieser Texte gehörten zu den Gipfel-Organisatoren.

In der von allen genannten Gremien und nicht zuletzt auch kürzlich vom Deutschen Ethikrat geforderten internationalen Ethikdebatte wird es neben Sicherheitsund Grenzziehungsfragen (das „Gespenst“ des Enhancement) auch um die immer wieder vorgebrachten kategorischen Verbotsargumente gehen müssen. Im Kern dieser Einwände steht zumeist die Vorstellung, der menschliche Genpool (,das Erbe der Menschheit“, wie die UNECSO mehrdeutig formuliert) dürfe nicht vorsätzlich verändert werden. Nun enthält dieser Genpool aber unzählige Variationen und ist zudem weder stabil noch durch einen ihn nobilitierenden Mechanismus entstanden. Die Konnotationen von Menschenwürdeverletzung und Menschheitsinteressen sind daher zumindest dort absurd, wo es sich um die Korrektur krankmachender Mutationen handelt.

Zusätzlich zu einer differenzierten und dynamischen, aber strikten nationalen Gesetzgebung muss die Wissenschaftsgemeinschaft neue Wege finden, um Abtrünnige wie He überzeugend zu ächten und um ihre Mitglieder, zumal ihren Nachwuchs, auf deren immense Verantwortung einzuschwören. Die Deklaration von Helsinki, forschungsethischer Kodex des Weltärztebundes mit 37 langen Paragraphen, ist das eine - ein Labor-Eid, der pathetisch zu schwören wäre, vielleicht das andere? Und auch über den wiederholt gemachten Vorschlag eines GLGE-Gremiums bei der WHO muss nachgedacht werden.

Interessenkonflikt B. Schöne-Seifert gibt an, dass kein Interessenkonflikt besteht. 


\section{Literatur}

Baltimore D, Charo A, Daley GQ et al (2018) On human genome editing II, statement by the Organizing Committee of the Second International Summit on human genome editing. http://www8. nationalacademies.org/onpinews/newsitem.aspx?RecordID=11282018b. Zugegriffen: 19. Dez. 2018

Dzau VJ, McNut M, Bai C (2018) Wake-up call from Hong Kong. Science 362(6420):1215. https://doi. org/10.1126/science.aaw3127

Epoch Times (2018) Genmanipulation an Babys unter Aufsicht der KP-Chinas? Chinesischer Forscher unter Hausarrest. Epoch Times 07.12.2018. https://www.epochtimes.de/china/china-medizin/ genmanipulation-an-babys-unter-aufsicht-der-kp-chinas-chinesischer-forscher-unter-hausarresta2731615.html. Zugegriffen: 19. Dez. 2018

Gaudelli NM, Komor AC, Rees HA et al (2017) Programmable base editing of $\mathrm{A} \bullet \mathrm{T}$ to G•C in genomic DNA without DNA cleavage. Nature 551(7681):464-471. https://doi.org/10.1038/nature24644

Middelton A, Niemiec E, Prainsack B et al (2018) 'Your DNA, Your Say': global survey gathering attitudes toward genomics: design, delivery and methods. Per Med 815(4):311-318. https://doi.org/10.2217/ pme-2018-0032

National Academies of Sciences, Engineering, and Medicine (2017) Human genome editing: science, ethics, and governance. National Academy Press, Washington DC

Nuffield Council (2018) Genome editing and human reproduction: social and ethical issues. Nuffield Council on Bioethics, London

Tang L, Zeng Y, Du H et al (2017) CRISPR/Cas9-mediated gene editing in human zygotes using Cas9 protein. Mol Genet Genomics. https://doi.org/10.1007/s00438-017-1299-z

Young E (2018) The CRISPR baby scandal gets worse by the day. The Atlantic 03.12.2018. https://www. theatlantic.com/science/archive/2018/12/15-worrying-things-about-crispr-babies-scandal/577234/.

Zugegriffen: 19. Dez. 2018 